\section{Tuberculosis among individuals with community-acquired pneumonia presenting to emer- gency in Gaborone, Botswana}

\author{
Jill K Gersh, ${ }^{1,2}$ Zachary Feldman, ${ }^{3,4}$ \\ Emily Greenberger, ${ }^{5,6}$ Amit Chandra, ${ }^{7}$ \\ Harvey M. Friedman, 5,6 \\ Ari Ho-Foster, ${ }^{5,6}$ Michelle K Haas ${ }^{5,6,8}$ \\ ${ }^{1}$ Division of Allergy and Infectious \\ Disease, Department of Medicine, \\ University of Washington, Seattle, WA, \\ USA; ${ }^{2}$ School of Medicine, University of \\ Colorado Anschutz Medical Campus, \\ Aurora, CO, USA; ${ }^{3}$ Department of \\ Economics, University of Colorado, \\ Boulder, CO, USA; ${ }^{4}$ Albers School of \\ Business and Economics, Seattle \\ University, Seattle, WA, USA; \\ ${ }^{5}$ Botswana UPenn Partnership, \\ Gaborone, Botswana; ${ }^{6}$ Division of \\ Infectious Disease, Department of \\ Medicine, Perelman School of Medicine, \\ University of Pennsylvania, \\ Philadelphia, PA, USA; ${ }^{7}$ Princess \\ Marina Hospital \& Department of \\ Emergency Medicine, University of \\ Botswana, School of Medicine, \\ Gaborone, Botswana; ${ }^{8}$ Denver Metro \\ Tuberculosis Program, Denver Public \\ Health, Denver, CO, USA
}

\begin{abstract}
Delays in diagnosing Tuberculosis (TB) are associated with increased transmission. TB may present as a clinical syndrome that mimics community-acquired pneumonia (CAP). The aim of this paper was to determine frequency of TB among patients with $\mathrm{CAP}$ at a referral hospital in Gaborone, Botswana. We performed a retrospective study of adults presenting with CAP from April 2010-October 2011 to the Emergency Department (ED); we matched this cohort to the National Botswana Tuberculosis Registry (NBTR) to identify individuals subsequently diagnosed with TB. We assessed demographics, time to TB diagnosis, clinical outcomes and performed logistic regressions to identify factors associated with TB diagnosis. We identified 1305 individuals presenting with CAP; TB was subsequently diagnosed in $68(5.2 \%)$. The median time to TB diagnosis was 9.5 days. Forty percent were AFB sputum smear positive and $87 \%$ were identified as being HIV-positive. Subsequent diagnosis of $\mathrm{TB}$ is common among individuals with CAP at our ED, sug-
\end{abstract}

gesting that TB may be present at the time of CAP presentation. Given the lack of distinguishing clinical factors between pulmonary $\mathrm{TB}$ and CAP, adults presenting with CAP should be evaluated for active $\mathrm{TB}$ in Botswana.

\section{Introduction}

Botswana remains a country heavily affected by tuberculosis (TB), with an estimated 2015 incidence of 356 per 100,000 in the population. ${ }^{1} \mathrm{~TB}$ has seen a resurgence in part due to the burden of the human immunodeficiency virus (HIV); roughly $60 \%$ of all individuals with $\mathrm{TB}$ in Botswana have underlying HIV. ${ }^{1}$ The diagnosis of HIV-related TB is particularly challenging as patients often present with acid-fast bacilli (AFB) smear negative disease. ${ }^{2}$ Prior studies have demonstrated that sputum smear-negative TB accounts for $22 \%$ of pulmonary TB in Botswana, and is consistent with the high prevalence of HIV in the area. ${ }^{3,4}$ Use of nucleic-acid amplification tests (NAAT) has enhanced the ability to diagnose HIV-TB, however challenges remain in widespread implementation. ${ }^{5}$

Marked delays in TB diagnosis risk exposure of close contacts, transmission of $\mathrm{TB}$ and worsened outcomes. ${ }^{6} \mathrm{~TB}$ may present as an acute respiratory illness, mimicking or occurring concomitantly with communityacquired pneumonia (CAP), which could contribute to diagnostic delays, increasing the likelihood of TB transmission. ${ }^{7}$ Indeed, TB represents the leading respiratory illness among hospitalized HIV-infected individuals in Botswana. ${ }^{8}$ Among those with a microbiologic diagnosis of CAP, one South African study found Mycobacterium tuberculosis (MTB) in $39.6 \%$ of sputa. In this South African cohort, MTB was the most common cause of CAP in both HIV-positive and HIVnegative patients. Among those with HIV, $26 \%$ of those who died had microbiologically confirmed TB. ${ }^{9}$

TB is not routinely considered a leading cause of CAP, so specific screening may not be performed. Identifying TB among patients presenting with respiratory illness affords the chance to appropriately target therapy and improve outcomes. In this study, we sought to identify opportunities to diagnose TB earlier. Our primary objective was to determine the frequency of TB among patients presenting with CAP to a large referral hospital in Gaborone, Botswana. The secondary objective was to define factors, including HIV status, associated with a diagnosis of TB in this setting.
Correspondence: Jill K. Gersh, University of Washington, Division of Allergy \& Infectious Diseases, 1959 NE Pacific St., Box 356423, Seattle, WA 98195, USA.

Tel.: +509.590.9052 - Fax: +206.616.3892. E-mail: jgersh@uw.edu

Key words: HIV, cough, sputum, diagnosis

Acknowledgments: This work would not have been possible without the efforts of the parent study team who, through their work with the ED nurses, medical officers and patients, sought to define the mortality associated with CAP in the context of underlying HIV disease. We would also like to thank David Horne for his thoughtful review of the manuscript.

The authors would like to acknowledge Thomas Lere, of the Botswana National Tuberculosis Programme, Gaborone, Botswana, for his assistance in BNTP data management as well.

Contributions: EG, AC, AHF, HMF and $\mathrm{MH}$, contributed to data collection and management. AHF contributed to data analysis. JKG, $\mathrm{ZF}$ and $\mathrm{MH}$ participated in all phases of the study.

Funding: none.

Conference presentation: Part of this work was presented at the 46th Union World Conference on Lung Health, December 2-6, 2015, Cape Town, South Africa.

Received for publication: 18 October 2017. Accepted for publication: 19 January 2018.

This work is licensed under a Creative Commons Attribution NonCommercial 4.0 License (CC BY-NC 4.0).

(C) Copyright J.K. Gersh et al., 2018

Licensee PAGEPress, Italy

Journal of Public Health in Africa 2018; 9:803 doi:10.4081/jphia.2018.803

\section{Materials and Methods}

\section{Study population}

We extracted data captured in the Emergency Department electronic database and hospital charts for patients presenting with CAP to Princess Marina Hospital (PMH), a 567-bed public tertiary hospital in Gaborone, Botswana. We included persons age 18 years or older, presenting from April 2010 through September 2011 with the diagnosis of CAP. We included all patients with diagnoses of pneumonia, communityacquired pneumonia, and Pneumocystis jirovecii (PCP) pneumonia, given that the last is a presumptive clinical diagnosis with a lack of reliable microbiologic certainty in Botswana. Confirmed diagnoses, with new chest radiograph findings, and presumed diagnoses, without chest radiography performed, were included, given the intermittent 
availability of chest radiography. Additional diagnoses of chest infection and lower respiratory tract infection were included if they satisfied the following criteria: i) at least one new chest symptom within the preceding two weeks, including new or worsened cough, shortness of breath, pleuritic chest pain, and ii) fever $\left(\mathrm{T}>38.0^{\circ} \mathrm{C}\right)$ or hypothermia $\left(\mathrm{T}<36.0^{\circ} \mathrm{C}\right)$. Repeated visits within 10 days were excluded.

The patients identified with CAP were matched to the National Botswana Tuberculosis Registry (NBTR) to identify individuals who were subsequently diagnosed with TB. Matches were made based upon identification (known as Omang) numbers or passport numbers when available, and manually by name, when identification numbers were not provided. Patients with a date of TB diagnosis in the NBTR prior to CAP diagnosis in the Emergency Department were excluded. Those with a missing data entry for TB treatment outcome were considered lost to follow up.

In patients who were diagnosed with CAP we assessed the following characteristics: demographics including age, sex, HIV status; chest radiograph findings (when available); hospital disposition; presenting symptoms including cough, fever, night sweats, shortness of breath, weight loss, fatigue, vomiting, hemoptysis; history of TB; time to TB diagnosis; sputum smear result. Laboratory data such as sputum culture were not routinely available for all subjects matched to the NBTR and sputum for bacterial cultures are not routinely collected. The primary outcome was registration of $\mathrm{TB}$ diagnosis within 90 days of presentation with CAP, as determined from the NBTR.

\section{Statistical analysis}

Demographic frequencies of those with CAP were calculated using STATA version $12 .{ }^{10}$ Chi-squared analyses were used to compare demographics across patients grouped by CAP with TB and CAP without TB. Logistic regression was used to compare sputum smear results and known HIV statuses using odds ratios (ORs) with confidence intervals (CIs). All statistical tests assessed significance at the $5 \%$ level.

This study was approved by the Institutional Review Boards of the University of Pennsylvania, the Botswana Ministry of Health, and Princess Marina Hospital.

\section{Results}

\section{Study population}

Of 37,053 adults presenting to the $\mathrm{PMH}$ Emergency Department from April 2010 through December 2011, 1516, or 4.1\%, were diagnosed with CAP (Figure 1). Of all individuals presenting with $\mathrm{CAP}, 14$ individuals either died in the Emergency Department or were deceased upon arrival. Two hundred and eleven were excluded from matching with the NBTR due to their presentation fewer than 90 days from the end of the study period, and 27 were excluded due to their presence in the NBTR prior to their CAP presentation. Of the remaining 1278 adults presenting with CAP, 68 , or $5.2 \%$, matched to the NTBR.

Of the 68 CAP-TB matched patients, the median age was 35 (range 18-81) (Table 1). Thirty-one (46\%) were female, and $36(53 \%)$ were male; one patient had missing data. Fifty (74\%) were HIV positive, seven $(10 \%)$ were HIV negative, and 11 (16\%) had unknown HIV status. In the Emergency Department, 14 individuals with HIV reported being on antiretroviral therapy (ART); NBTR records show 17 were taking ART while 9 were not. Of 61 patients who had chest radiography performed, 45 (74\%) had positive chest $\mathrm{X}$-ray findings, which included consolidations, opacities, effusions, and infiltrates, while $67 \%$ of individuals with CAP without TB had a positive chest $\mathrm{x}$-ray. The disposition of the TB-CAP group was 34 admitted and 34 discharged.

Among the CAP-TB group matched to the NBTR, cough $(77 \%)$ was the most common symptom. The frequency of symptoms was similar across both the matched and unmatched groups, except the matched group

Table 1. Demographics of community-acquired pneumonia (CAP) presentations.

\begin{tabular}{lccc} 
& $\begin{array}{c}\text { CAP-TB } \\
(\mathrm{n}=68), \mathbf{n}(\%)\end{array}$ & $\begin{array}{c}\text { CAP without TBB } \\
(\mathrm{n}=1210), \mathrm{n}(\%)\end{array}$ & $\begin{array}{c}\text { All }(\mathrm{n}=1278), \\
\mathbf{n}(\%)\end{array}$ \\
Median Age & 35 & 38 & 37 \\
HIV Status & $50(73.5)$ & $780(59.8)$ & $760(59.5)$ \\
Positive & $7(10.3)$ & $113(8.7)$ & $109(8.5)$ \\
Negative & $11(16.2)$ & $412(31.6)$ & $409(32.0)$ \\
Unknown & $61(89.7)$ & $1016(84.0)$ & $1077(84.3)$ \\
\hline CXR ordered & $45 / 61(73.8)$ & $680 / 1016(66.9)$ & $725 / 1077(67.3)$ \\
Positive Chest Radiograph Finding* & & & \\
Disposition & & & \\
Admitted & $34(50.0)$ & $661 / 1186(55.7)$ & $695 / 1254(55.4)$ \\
Discharged & $34(50.0)$ & $511 / 1186(43.1)$ & $545 / 1254(43.5)$ \\
Cough & $52(76.5)$ & $875(72.3)$ & $927(72.5)$ \\
\hline Shortness of breath & $33(48.5)$ & $675(55.8)$ & $708(55.4)$ \\
Night sweats & $20(29.4)$ & $208(17.2)$ & $228(17.8)$ \\
\hline Fatigue & $11(16.2)$ & $195(16.1)$ & $206(16.1)$ \\
Fever & $23(33.8)$ & $362(29.9)$ & $385(30.1)$ \\
\hline Weight loss & $9(13.2)$ & $110(9.1)$ & $119(9.3)$ \\
Vomiting & $9(13.2)$ & $208(17.2)$ & $217(17.0)$ \\
\hline Hemoptysis & $9(13.2)$ & $85(7.0)$ & $94(7.4)$ \\
Known history of TB & $19(28.0)$ & $265(21.9)$ & $284(22.2)$ \\
\hline
\end{tabular}

*Denominator reflects number of radiographs ordered. ${ }^{\circ}$ Denominator reflects exclusion of missing disposition record had a significantly higher frequency of night sweats (P-value 0.01). The matched group also had higher frequencies of hemoptysis (Pvalue 0.056 ) and weight loss (P-value 0.252) although these did not reach statistical significance (Table 1). Nineteen $(28 \%)$ patients with a subsequent TB diagnosis, and 265 $(22 \%)$ of those without, knew their prior history of TB disease.

\section{TB diagnosis}

Among the matched CAP-TB group, 27 (40\%) were sputum smear positive, $17(25 \%)$ were sputum smear negative, 24 (35\%) had unknown or missing sputum smear results. The median time from ED visit to diagnosis was 9.5 days. Four (6\%) patients were diagnosed with TB the same day as presenting with CAP by AFB smear; 43 (63\%) were diagnosed within 15 days of CAP presentation, and 60 (88\%) within 90 days (Figure 2). The difference in time-to-diagnosis between the 27 smear positive patients and the 41 smear negative or unknown patients was 1 day (median 10 days for smear positive, 9 days for smear negative/unknown). When comparing above and below the median age, those 37 years and older were less likely to be diagnosed with TB within the study period [OR $0.504(95 \%$ CI $0.301,0.844)]$. Of the 68 individuals who matched in the CAP-TB group, all were started on TB treatment per the NBTR records. Of the 16 whose treatment outcome was known from the NBTR records, 14 completed treatment, one defaulted on treatment, and one patient died. The 
remaining 52 patients who started on treatment per the National TB Registry were lost to follow up.

\section{HIV status}

Eighty-four percent of those in the CAPTB group had a known HIV status. Both those with subsequent $\mathrm{TB}$ diagnoses and those without had an HIV positive rate of $87 \%$. HIV negative individuals had an $83 \%$ smear positive rate, compared to approximately $50 \%$ smear positive rate among those with HIV. More people with HIV had sputum samples performed (29 samples) compared to HIV negative individuals (6 samples). Those with known HIV status were more likely to be diagnosed with TB within 90 days compared to those with unknown status [OR 3.36 (95\% CI 1.51, 7.48)].

\section{Discussion}

Derived from one of the largest samples of patients with CAP in sub-Saharan Africa, this study highlights the challenges in screening for TB in acute care settings and demonstrates opportunities for missed diagnosis in prevalent areas. Five percent of patients presenting with CAP were later registered in the National TB registry, representing a cohort of individuals who experienced a delay in diagnosis, risking transmission of TB to their families, in healthcare facilities and within the community. Not surprisingly, the majority of patients in our cohort also had underlying HIV and were more likely to present with smear-negative disease. The presence of HIV can further limit the diagnostic certainty of TB screening, as seen among these patients with a high HIV prevalence (87\%) in a country with an HIV prevalence of $22 \% .^{11}$ Our study demonstrates the lack of distinguishing clinical features between patients presenting with CAP and those with an underlying diagnosis of TB. There were no significant differences between the CAP-TB group and the CAP group regarding vital signs, respiratory symptoms, weight loss or night sweats. This supports the low specificity and known limitations in diagnostic value of a clinical symptom assessment, such as the WHO symptom screen, in HIV endemic areas. ${ }^{12,13}$ In HIV care settings, the proportion of subclinical TB (sputum-culture positive with negative symptom screen) can approach $15 \%$ of new diagnoses. ${ }^{13}$ In an acute care setting, such as the Emergency Department, when alternative diagnoses are assigned based upon overlapping syndromes or negative symptom screens, subclinical TB may go undiagnosed in favor of another clinical diagnosis and timely initiation of therapy. As seen in a South African study of the microbiological diagnoses of CAP, M. tuberculosis was the most common pathogen isolated; however a microbiological diagnosis is infrequently available in clinical settings. ${ }^{9}$ The line between CAP and TB can become blurred, especially in the context of HIV infection.

The Botswana antimicrobial guidelines recognize TB as a common cause of CAP and encourage sputum collection for AFB smear from those presenting with cough of greater than one week and all HIV positive individuals with concern for CAP. ${ }^{14}$ A third of patients presenting with CAP had unknown or missing sputum smear results, highlighting the challenges faced by patients and their providers in resource-constrained settings. Limitations in collecting sputum and long laboratory delays in releasing sputum smear results have been cited by Batswana physicians as obstacles to full implementation of the national TB guidelines. ${ }^{3}$ This leads to a reliance on alternative diagnoses, tests, or empiric therapy in settings with a high proportion of smear-unknown or smear-negative TB, such as in HIV prevalent areas. ${ }^{3}$ Negative sputum smear results can lead to delays in diagnosis or omission from further work up. ${ }^{3,4}$ The WHO's 2010 endorsement of the Xpert MTB/RIF assay for TB investigation was a step towards an enhanced screening algorithm. Xpert MTB/RIF was successfully implemented in Botswana in 2011; however with an unsuccessful test incidence of up to $15 \%$, continued efforts in training and improved sampling are needed. ${ }^{16}$ Early studies found that in populations with high smear-negative rates of TB, such as HIVprevalent populations, screening with Xpert increased the case detection rate by $45 \%$ compared to smear microscopy. ${ }^{5,15}$ Since the

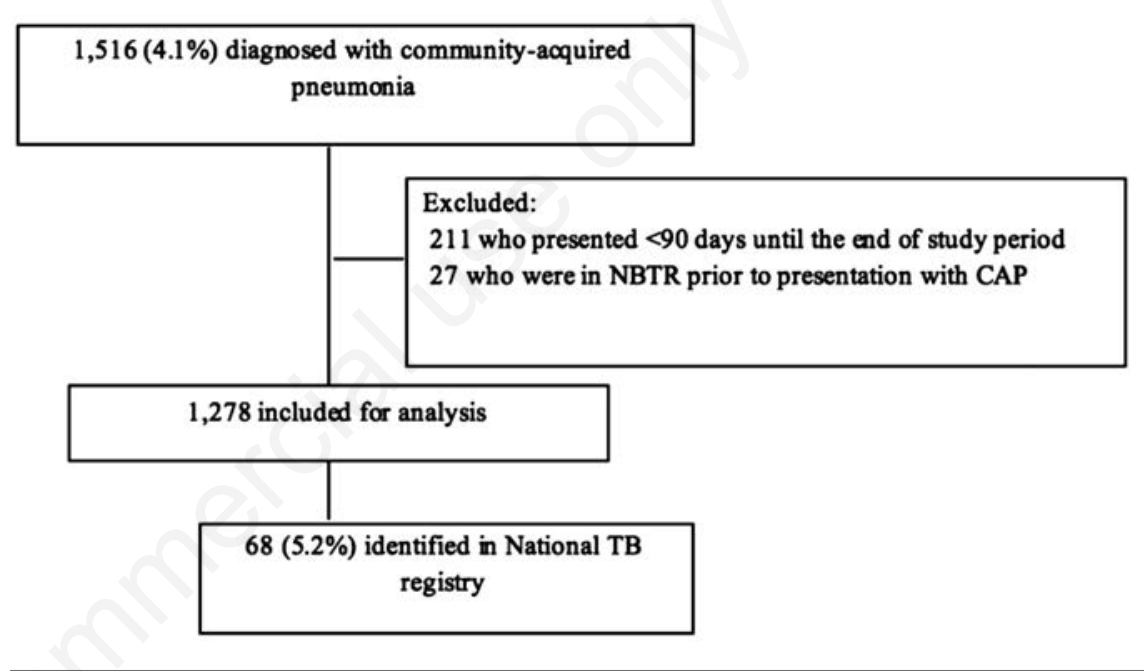

Figure 1. Study cohort collection.

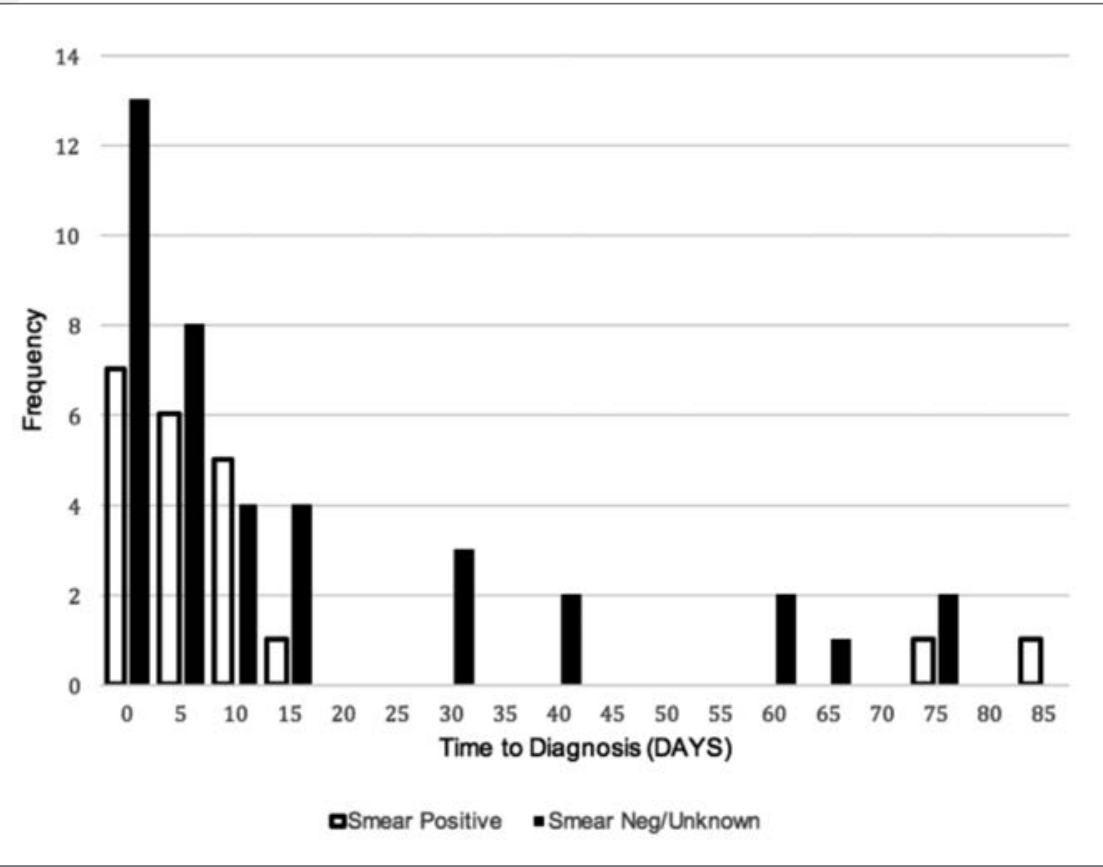

Figure 2. Time to diagnosis. 
roll out of Xpert, studies in Botswana have shown that greater than half of positive sputum cultures among people living with HIV with a positive WHO symptom screen show non-tuberculous mycobacteria (NTM), demonstrating a significant limitation with reliance upon smear microscopy for diagnosis of TB, such as in our study. ${ }^{17}$ Furthermore, our findings that $\mathrm{TB}$ was diagnosed no sooner with positive sputum smear raises concern for potential problems along the TB care cascade, such as laboratory delays, paucity of sputum sample collection, or clinician anchoring bias to CAP diagnosis.

HIV prevalence was high $(87 \%)$ in CAP patients with subsequent TB and those without. More patients with HIV had sputum samples collected compared to those without HIV (29 samples vs 6 samples), which is likely related to an appropriately heightened clinical suspicion for TB among people living with HIV. ${ }^{4,12,13,18}$ However, we saw a trend of positive smear results occurring more commonly in HIV negative individuals ( $83 \%$ smear positive rate) compared to HIV positive individuals ( $48 \%$ smear positive rate), supporting the often paucibacillary nature of HIV-associated TB. ${ }^{19}$ Importantly, there was no difference in time to diagnosis for individuals with negative AFB smears compared to those with positive AFB smears, highlighting the delays in laboratory testing, processing of smear positive samples, and diagnosis among co-infected persons. While the effort to test for TB in HIV may be well intended, the diagnostic value of sputum smear is limited in HIV disease, and may contribute to the rate of false negative TB testing in HIV. $4,9,16$

Our study was limited by the single site and retrospective study design. Charts often had incomplete or missing data, as seen with $35 \%$ of patients with missing or unknown sputum smear results. The study selected for those well enough to present to care for subsequent TB diagnosis after their Emergency Department visit. Those with chronic symptoms, which are more likely in TB disease, may not present to the Emergency Department, thus our sample may be an underestimation of TB disease presenting as CAP. ${ }^{12}$ Furthermore we did not examine Emergency Department records for patients without CAP symptoms who nevertheless underwent evaluation for TB disease. Per the Botswana National TB Program 2011 guidelines, TB screening should include those with a history or new diagnosis of HIV. ${ }^{20}$ Patients presenting to the Emergency Department with syndromes consistent with advanced HIV without CAP criteria were not included in our study. We suspect that our study underestimated the true proportion of missed TB diagnoses at this Emergency Department.

\section{Conclusions}

The confirmation of TB following presentations with CAP highlights opportunities for missed TB diagnoses in acute care settings in high TB burden communities. Undiagnosed TB impacts the morbidity and mortality of individuals and the health of the community through unchecked transmission. Harnessing additional points of health care access to evaluate for $\mathrm{TB}$, in the context of concurrent clinical syndromes, can positively affect the TB care cascade and the health of the community.

\section{References}

1. WHO. Global Tuberculosis Report 2016. Available from: http://scholar.google.com/scholar?hl=en\& btnG $=$ Search\&q=intitle:No+Title $\# 0 \% 0 \mathrm{Ah}$ ttp://scholar.google.com/scholar?hl=en\&bt $\mathrm{nG}=$ Search\&q=intitle:No+title $\# 0$

2. Dickinson DB. Facilitating point-of-care detection/suspicion of early TB disease to enable early treatment access, while awaiting more definitive microbiologic diagnosis. J Int AIDS Soc 2016;19:19-21.

3. Tafuma TA, Burnett RJ, Huis In't Veld D. National guidelines not always followed when diagnosing smear-negative pulmonary tuberculosis in patients with HIV in Botswana. PLoS One 2014;9:1-5.

4. Talbot E, Hay Burgess DC, Hone NM, et al. Tuberculosis serodiagnosis in a predominantly HIV-infected population of hospitalized patients with cough, Botswana, 2002. Clin Infect Dis 2004;39:e1-7.

5. Lawn SD, Mwaba P, Bates $M$, et al. Advances in tuberculosis diagnostics: The Xpert MTB/RIF assay and future prospects for a point-of-care test. Lancet Infect Dis 2013;13:349-61.

6. Asch S, Leake B, Anderson R, Gelberg L. Care for Tuberculosis? Am J Respir Crit Care Med 1998;157:1244-8.

7. Feng JY, Fang WF, Wu CL, et al. Concomitant pulmonary tuberculosis in hospitalized healthcare-associated pneumonia in a tuberculosis endemic area: A multi-center retrospective study. PLoS One 2012;7:1-9.

8. Lockman S, Hone N, Kenyon TA, et al. Etiology of pulmonary infections in predominantly HIV-infected adults with suspected tuberculosis, Botswana. Int J Tuberc Lung Dis 2003;7:714-23.

9. Nyamande K, Lalloo UG, John M. TB presenting as community-acquired pneumonia in a setting of high TB incidence and high HIV prevalence. Int J Tuberc Lung Dis 2007;11:1308-13.
10. StataCorp. Stata Statistical Software: Release 12. College State, TX: StataCorp LP; 2011.

11. UNAIDS. AIDSinfo 2015. Available from: http://aidsinfo.unaids. org?did\%3D5581277ae9beccab3bd5a44e $\% 26 r \% 3 D w o r l d \% 26 t \% 3 D 2015 \% 26 t b \% 3$ Dq\%26bt $\% 3$ Dundefined $\% 26$ ts $\% 3$ Dnull $\%$ $26 \mathrm{qla} \% 3 \mathrm{DC} \% 26 \mathrm{qls} \% 3 \mathrm{DBWA}$

12. Getahun H, Kittikraisak W, Heilig CM, et al. Development of a standardized screening rule for tuberculosis in people living with HIV in resource-constrained settings: Individual participant data meta-analysis of observational studies. PLoS Med 2011;8.

13. Corbett EL, MacPherson P. Tuberculosis screening in high human immunodeficiency virus prevalence settings: Turning promise into reality. Int J Tuberc Lung Dis 2013;17:1125-38.

14. Ministry of Health. Botswana antimicrobial therapy guide. 2012. Available from: https://aidsfree.usaid. gov/sites/default/ files/tx_botswana_2012.pdf

15. Lawn SD, Brooks SV, Kranzer K, et al. Screening for HIV-associated tuberculosis and rifampicin resistance before antiretroviral therapy using the Xpert MTB/RIF assay: A prospective study. PLoS Med 2011;8.

16. Agizew T, Boyd R, Ndwapi N, et al. Peripheral clinic versus centralized laboratory-based Xpert MTB/RIF performance: Experience gained from a pragmatic, stepped-wedge trial in Botswana. PLoS One 2017;12:1-14.

17. Agizew T, Basotli J, Alexander $\mathrm{H}$, et al. Higher-than-expected prevalence of nontuberculous mycobacteria in HIV setting in Botswana: Implications for diagnostic algorithms using Xpert MTB/RIF assay. PLoS One 2017;12:1-13.

18. Burmen B, Modi S, Cavanaugh JS, et al. Tuberculosis screening outcomes for newly diagnosed persons living with HIV, Nyanza Province, Kenya, 2009. Int J Tuberc Lung Dis 2016;20:79-84.

19. Montales MT, Chaudhury A, Beebe A, et al. HIV-Associated TB Syndemic: A Growing Clinical Challenge Worldwide. Front Public Heal 2015;3:1-7.

20. Ministry of Health Botswana, WHO, African Comprehensive HIV and AIDS Partnerships (ACHAP), Centers for Disease Control and Prevention (CDC/BOTUSA), KNCV Netherlands Tuberculosis Foundation, (BUP) B-UP. Botswana TB/HIV policy guidelines. 2011. 